\title{
Light-Quark Resonances at COMPASS
}

\author{
Stefan Wallner ${ }^{\mathrm{ab}}$ \\ Institute for Hadronic Structure and Fundamental Symmetries (TU Munich) \\ E-mail: stefan.wallneretum.de
}

The main goal of the spectroscopy program at COMPASS is to explore the light-meson spectrum in the mass range below about $2 \mathrm{GeV} / c^{2}$ using diffractive dissociation reactions. Our flagship channel is the production of three charged pions in the reaction: $\pi^{-}+p \rightarrow \pi^{-} \pi^{-} \pi^{+}+p_{\text {recoil }}$, for which COMPASS has acquired the so far world's largest dataset of roughly $50 \mathrm{M}$ exclusive events using an $190 \mathrm{GeV} / c \pi^{-}$beam. Based on this dataset, we performed an extensive partial-wave analysis.

In order to extract the parameters of the $\pi_{J}$ and $a_{J}$ resonances that appear in the $\pi^{-} \pi^{-} \pi^{+}$ system, we performed the so far most comprehensive resonance-model fit, using Breit-Wigner parametrizations. This method in combination with the high statistical precision of our data allows us to study ground and excited states. We study the $a_{4}(2040)$ resonance in the $\rho(770) \pi G$ and $f_{2}(1270) \pi F$ decays. In addition to the ground state resonance $a_{1}(1260)$, we have found evidence for the $a_{1}(1640)$, which is the first excitations of the $a_{1}(1260)$, in our data. We also study the spectrum of $\pi_{2}$ states by simultaneously describing four $J^{P C}=2^{-+}$waves using three $\pi_{2}$ resonances, the $\pi_{2}(1670)$, the $\pi_{2}(1880)$, and the $\pi_{2}(2005)$.

Using a novel analysis approach, where the resonance-model fit is performed simultaneously in narrow bins of the squared four-momentum transfer $t^{\prime}$ between the beam pion and the target proton, allows us to study the $t^{\prime}$ dependence of resonant and non-resonant components included in our model. We observe that for most of the partial waves, the non-resonant components show a steeper $t^{\prime}$ spectrum compared to the resonances and that the $t^{\prime}$ spectrum of most of the resonances becomes shallower with increasing resonance mass. We also study the $t^{\prime}$ dependence of the relative phases between resonance components. The pattern we observe is consistent with a common production mechanism of these states.

XIII Quark Confinement and the Hadron Spectrum - Confinement2018

31 July - 6 August 2018

Maynooth University, Ireland

\footnotetext{
${ }^{\text {a }}$ Speaker.

${ }^{b}$ For the COMPASS collaboration
} 


\section{Light-meson spectroscopy at COMPASS}

COMPASS is a fixed-target multi-purpose experiment located at CERN. Positive and negative secondary hadron beams or a tertiary muon beam are directed onto various types of targets. The forward-going final-state particles are detected by a two-stage magnetic spectrometer, which has a large acceptance over a broad kinematic range.

The main goal of the COMPASS spectroscopy program is to study the light-meson spectrum up to masses of about $2 \mathrm{GeV} / c^{2}$. At COMPASS, these light mesons are produced in diffractive scattering of the $190 \mathrm{GeV} / c$ negative hadron beam, which contains mainly negative pions, off a liquid-hydrogen target. In these reactions, we can study $a_{J}$ and $\pi_{J}$-like mesons. Due to their very short lifetime, we observe the resonances only in their decays into quasi-stable final-state particles. Our flagship channel is the decay into three charged pions: $\pi^{-}+p \rightarrow \pi^{-} \pi^{-} \pi^{+}+p_{\text {recoil }}$. COMPASS has acquired the so far world's largest dataset of about $50 \mathrm{M}$ exclusive events for this channel, which allows us to apply novel analysis methods [1].

\section{Analysis method}

\subsection{Partial-wave decomposition}

We employ the method of partial-wave analysis in a two-step approach. In the first step, called partial-wave decomposition, data are decomposed into contributions from various partial waves [1]. To this end, we construct a model for the intensity distribution $\mathscr{I}(\tau)$ of the $\pi^{-} \pi^{-} \pi^{+}$final state in terms of the five-dimensional phase-space variables of the $3 \pi$ system that are represented by $\tau$. Using the isobar approach, $\mathscr{I}(\tau)$ is modeled as a coherent sum of partial-wave amplitudes, which are defined by the quantum numbers of the $3 \pi$ system $\left(J^{P C} M^{\varepsilon}\right)$, ${ }^{[a]}$ the intermediate $\pi^{-} \pi^{+}$ resonance $\zeta$ through which the decay proceeds, and the orbital angular momentum $L$ between the bachelor pion and the isobar. These quantum numbers are represented by $a=J^{P C} M^{\varepsilon} \zeta \pi L$ :

$$
\mathscr{I}\left(\tau ; m_{3 \pi}, t^{\prime}\right)=\left|\sum_{a}^{\text {waves }} \mathscr{T}_{a}\left(m_{3 \pi}, t^{\prime}\right) \psi_{a}\left(\tau ; m_{3 \pi}, t^{\prime}\right)\right|^{2} .
$$

Within the isobar model, the decay amplitudes $\psi_{a}$ can be calculated. This allows us to extract the partial-wave amplitudes $\mathscr{T}_{a}$, which determine the strength and phase of each wave, from the data by an unbinned maximum-likelihood fit.

In order to extract the $m_{3 \pi}$ dependence of the partial-wave amplitudes, the maximum-likelihood fit is performed independently in narrow bins of the three-pion mass $m_{3 \pi}$. In addition, the large size of the dataset allows us to study the dependence of the partial-wave amplitudes on the squared four-momentum transfer $t^{\prime}$, by binning the data in $t^{\prime}$ as well. By performing the partial-wave decomposition independently in $100 m_{3 \pi}$ bins in the range $0.5<m_{3 \pi}<2.5 \mathrm{GeV} / c^{2}$ and $11 t^{\prime}$ bins in the range $0.1<t^{\prime}<1.0(\mathrm{GeV} / c)^{2}{ }^{[\mathrm{b}]}$ we extract simultaneously the $m_{3 \pi}$ and $t^{\prime}$ dependence of the partial-wave amplitudes from the data.

We use a wave-set of 88 partial waves with spin $J$ and angular momentum $L$ up to six, which includes six different isobars. This is the largest wave-set used so far for this channel.

\footnotetext{
${ }^{[a]}$ Here, $J$ is the spin of the $3 \pi$ state, $P$ its parity and $C$ its charge conjugation quantum number. The spin projection of $J$ along the beam axis is given by $M^{\varepsilon}$.

${ }^{[b]}$ By definition, $t^{\prime}$ is a positive quantity, as $t^{\prime} \equiv|t|-|t|_{\min }$.
} 


\subsection{Resonance-model fit}

In the second step of this analysis, the so-called resonance-model fit, we parameterize the $m_{3 \pi}$ dependence of the partial-wave amplitudes in order to extract the masses and widths of resonances appearing in the $\pi^{-} \pi^{-} \pi^{+}$system. Details can be found in ref. [2]. We model the amplitude for a wave $a$ as a coherent sum over wave components $j$ that we assume to contribute to this wave: ${ }^{[c]}$

$$
\mathscr{T}_{a}\left(m_{3 \pi}, t^{\prime}\right)=\sum_{j \in \mathbb{S}_{a}} \mathscr{T}_{a}^{j}\left(m_{3 \pi}, t^{\prime}\right)=\sum_{j \in \mathbb{S}_{a}} \mathscr{C}_{a}^{j}\left(t^{\prime}\right) \cdot \mathscr{D}^{j}\left(m_{3 \pi}, t^{\prime} ; \zeta_{j}\right)
$$

The dynamical amplitudes $\mathscr{D}^{j}\left(m_{3 \pi}, t^{\prime} ; \zeta_{k}\right)$ represent the resonant and non-resonant wave components. We use relativistic Breit-Wigner amplitudes for resonances and a phenomenological parameterization for the non-resonant components. ${ }^{[\mathrm{d}]}$ Each dynamical amplitude is multiplied by a coupling amplitude $\mathscr{C}_{a}^{j}\left(t^{\prime}\right)$, which determines the strength and phase with which each wave component $j$ contributes to the corresponding wave $a$. We use independent coupling amplitudes for each $t^{\prime}$ bin. Thus, also in this analysis step, we do not impose a model for the $t^{\prime}$ dependence of the amplitudes of the wave components. We simultaneously describe all $11 t^{\prime}$ bins in a single resonance-model fit, keeping the mass and width parameters of each resonance component the same in each $t^{\prime}$ bin. In this way, our $t^{\prime}$-resolved analysis gives us an additional dimension of information, which helps to better separate resonant from non-resonant components. Furthermore, it allows us to extract the $t^{\prime}$ dependence of each wave component in different partial waves as discussed below.

Our two-step approach allows us to select a subset of waves to be included in the resonancemodel fit. In this analysis, we select a subset of 14 out of the 88 partial waves with $J^{P C}=0^{-+}$, $1^{++}, 1^{-+}, 2^{++}, 2^{-+}$, and $4^{++}$quantum numbers. This subset accounts for about $60 \%$ of the total intensity. The 14 waves are parameterized by 11 resonances: $\pi(1800), a_{1}(1260), a_{1}(1420)$, $a_{1}(1640), \pi_{1}(1600), a_{2}(1320), a_{2}(1700), \pi_{2}(1670), \pi_{2}(1880), \pi_{2}(2005)$, and $a_{4}(2040)$ plus a nonresonant component in each wave.

After separating the individual resonant and non-resonant components in the resonance-model fit, we can determine the $t^{\prime}$ dependence of their intensities, i.e. the $t^{\prime}$ spectra, and the $t^{\prime}$ dependence of the relative phases of the coupling amplitudes. Integrating the intensity over $m_{3 \pi}$ gives the $t^{\prime}$ dependent yield, i.e. the $t^{\prime}$ spectrum, which is the number of events of wave component $j$ in wave $a$ in the $11 t^{\prime}$ bins:

$$
\mathscr{I}_{a}^{j}\left(t^{\prime}\right)=\frac{1}{\Delta t^{\prime}} \int_{m_{\min }}^{m_{\max }} \mathrm{d} m_{3 \pi}\left|\mathscr{T}_{a}^{j}\left(m_{3 \pi}, t^{\prime}\right)\right|^{2} .
$$

To account for the non-equidistant $t^{\prime}$-binning, we normalize in each $t^{\prime}$ bin the intensity to the respective bin width $\Delta t^{\prime}$. The intensity of most wave components falls approximately exponentially with increasing $t^{\prime}$. This is consistent with the expectation from Regge theory. For waves with spin projection $M \neq 0$, the intensity is kinematically suppressed for small values of $t^{\prime}$ by an additional $\left(t^{\prime}\right)^{|M|}$ factor [3]. Therefore, we fit the model

$$
\mathscr{I}_{a}^{j}\left(t^{\prime}\right)=A_{a}^{j} \cdot\left(t^{\prime}\right)^{|M|} \cdot e^{-b_{a}^{j} t^{\prime}}
$$

\footnotetext{
${ }^{[c]}$ For simplicity, we absorb here the $\sqrt{m_{3 \pi}}$ factor, the phase space integral, and the production factor in the dynamical amplitudes (see ref. [2] for details).

${ }^{[\mathrm{d}]}$ The leading $m_{3 \pi}$ dependence of the non-resonant term is $e^{-c \tilde{q}^{2}\left(m_{3 \pi}\right)}$, where $\tilde{q}\left(m_{3 \pi}\right)$ is an approximation for the two-body break-up momentum of the isobar pion system, which is also valid below threshold.
} 
to the $t^{\prime}$ spectra extracted from the resonance-model fit. The real-valued parameter $A_{a}^{j}$ and the slope parameter $b_{a}^{j}$ are left free in the fit. Thereby, we extract one slop parameter for each wave component $j$ and for each partial wave $a$ that includes this wave component.

Special cases are resonance components that appear in different partial waves with the same $J^{P C} M^{\varepsilon}$ quantum numbers, but different decay modes $\zeta \pi L$. As the $t^{\prime}$ dependence is a property of the production and not of the decay, the same resonance component in different decay modes should show the same $t^{\prime}$ dependence. We incorporate this constraint into our model by fixing the $t^{\prime}$ dependence $\mathscr{C}_{b}^{j}\left(t^{\prime}\right)$ of a resonance $j$ that appears in wave $b$ to the $t^{\prime}$ dependence $\mathscr{C}_{a}^{j}\left(t^{\prime}\right)$ in a reference wave $a$ via

$$
\mathscr{C}_{b}^{j}\left(t^{\prime}\right)={ }_{b} \mathscr{B}_{a}^{j} \mathscr{C}_{a}^{j}\left(t^{\prime}\right)
$$

The $t^{\prime}$-independent complex-valued branching amplitude $\mathscr{B}_{a}^{j}$ represents the relative strength and phase between the two decay modes and is the only remaining free parameter for resonance component $j$ in wave $b$. By this constraint, the $t^{\prime}$ spectra of resonance $j$ in waves $a$ and $b$ are the same, except for a $t^{\prime}$-independent scaling factor proportional to $\left|{ }_{b} \mathscr{B}_{a}^{j}\right|^{2}$. [e] Also, the $t^{\prime}$ dependence of the phase of resonance $j$ in waves $a$ and $b$ is the same, except for the $t^{\prime}$-independent phase offset $\arg \left[\mathscr{B}_{a}^{j}\right]$.

The fit result is affected by many systematic effects, e.g. the choice of the parameterizations of the wave components, the selected 14-wave sub-set, or the constraints imposed by equation (2.5). We performed more than 200 systematic studies to improve our model, to study the evidence for some resonance signals, and to determine the systematic uncertainties of the extracted parameters.

\section{Selected Results of the resonance-model fit}

In the following subsections, we present selected results for resonances with $J^{P C}=4^{++}, 1^{++}$, and $2^{-+}$quantum numbers. As the statistical uncertainties are at least one order of magnitude smaller than the systematic ones, we quote only systematic uncertainties. A more exhaustive discussion of the results of the resonance-model fit can be found in ref. [2]. 1

\section{1 $\mathrm{J}^{P C}=4^{++}$resonances}

Figure 1a shows the intensity distribution of the $4^{++} 1^{+} \rho(770) \pi G$ wave summed over all $11 t^{\prime}$ bins ( $t^{\prime}$-summed). It exhibits a clear peak at about $2 \mathrm{GeV} / c^{2}$, which is nearly completely described by the $a_{4}(2040)$ resonance component. The relative phase of the $4^{++} 1^{+} \rho(770) \pi G$ wave with respect to, e.g., the $1^{++} 0^{+} \rho(770) \pi S$ wave is shown in figure 1c. We observe a clear rise in the $2 \mathrm{GeV} / c^{2}$ mass region, as expected for a resonance. The low-mass tail of the peak is described as an interference effect between the $a_{4}(2040)$ and the non-resonant component in this wave. Also in the $4^{++} 1^{+} f_{2}(1270) \pi F$ wave, we observe a clear $a_{4}(2040)$ signal as shown in figure $1 \mathrm{~b}$. Our estimates for the $a_{4}(2040)$ resonance parameters are $m_{0}=1935_{-13}^{+11} \mathrm{MeV} / c^{2}$ and $\Gamma_{0}=333_{-21}^{+16} \mathrm{MeV} / c^{2}$. They are comparably robust with respect to systematic effects, due to the small non-resonant contributions in these $4^{++}$waves. Our estimates for the $a_{4}(2040)$ mass and

\footnotetext{
${ }^{[\mathrm{e}]}$ In addition to $\left|{ }_{b} \mathscr{B}_{a}^{j}\right|^{2}$, the different phase-space integrals enter the scaling factor. Furthermore, the production amplitude causes a slight $t^{\prime}$ dependence of this factor, which we drop here. See ref. [2] for details.
} 


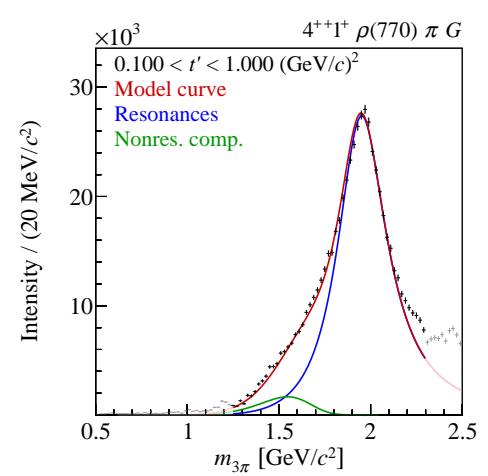

(a)

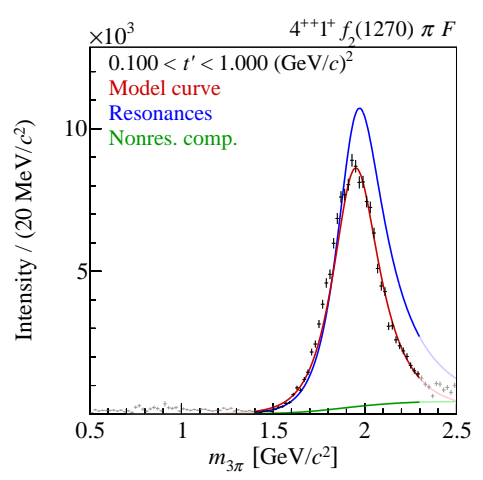

(b)

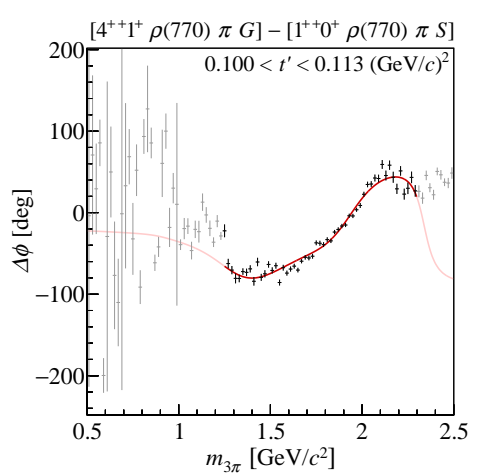

(c)

Figure 1: $t^{\prime}$-summed intensity distribution of (a) the $4^{++} 1^{+} \rho(770) \pi G$ wave and (b) the $4^{++} 1^{+}$ $f_{2}(1270) \pi F$ wave. (c) shows the relative phase of the $4^{++} 1^{+} \rho(770) \pi G$ wave with respect to the $1^{++} 0^{+} \rho(770) \pi S$ wave in the lowest $t^{\prime}$ bin. The data points show the result of the partial-wave decomposition. Uncertainties are statistical only. The red curves represent the resonance model. The blue curves represent the $a_{4}(2040)$ resonance. The green curves represent the non-resonant components. The extrapolations beyond the fitted $m_{3 \pi}$ range are shown in lighter colors.

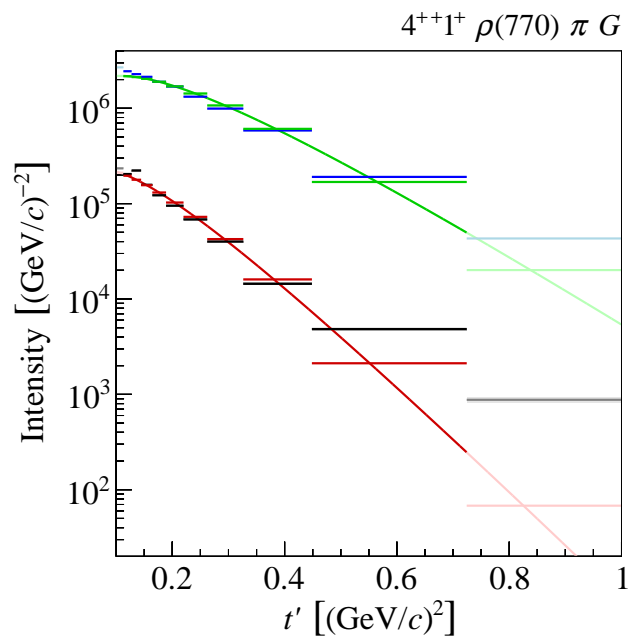

(a)

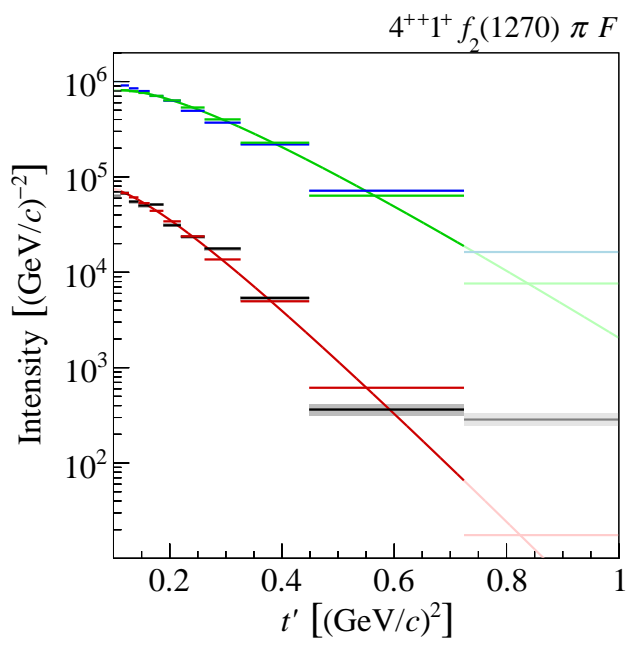

(b)

Figure 2: $t^{\prime}$ spectra of the $a_{4}(2040)$ and the non-resonant components in (a) the $4^{++} 1^{+} \rho(770) \pi G$ wave and (b) the $4^{++} 1^{+} f_{2}(1270) \pi F$ wave. The black horizontal lines indicate the central values and the gray boxes the statistical uncertainties of the $t^{\prime}$ spectra of the non-resonant component. The blue lines and boxes represent the $t^{\prime}$ spectra of the $a_{4}(2040)$. The red and green curves and lines show the results of a fit of equation (2.4) to these data. 


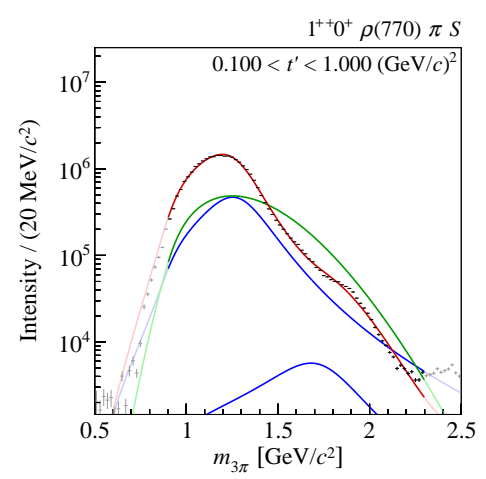

(a)

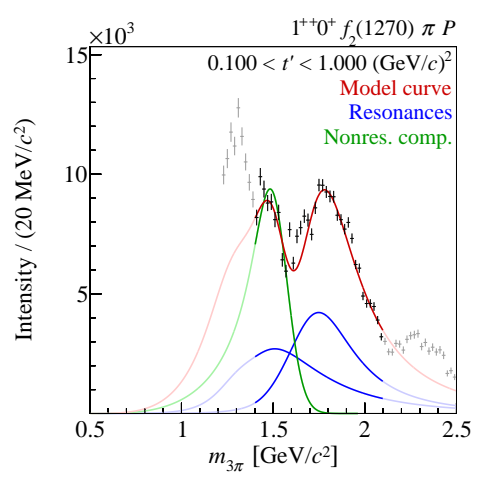

(b)

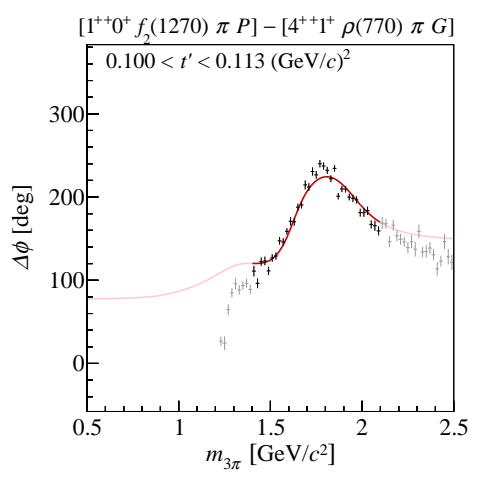

(c)

Figure 3: $t^{\prime}$-summed intensity distributions of (a) the $1^{++} 0^{+} \rho(770) \pi S$ and (b) the $1^{++} 0^{+}$ $f_{2}(1270) \pi P$ wave. (c) shows the relative phase of the $1^{++} 0^{+} f_{2}(1270) \pi P$ wave with respect to the $4^{++} 1^{+} \rho(770) \pi G$ wave in the lowest $t^{\prime}$ bin. Same color code as in figure 1 is used.

width agree with previous measurements, based on diffractive production $[4,5,6,7]$. However, our values is at variance with other measurements, e.g. from production in $\bar{p} p$ collisions [8].

Including both, the $\rho(770) \pi$ and the $f_{2}(1270) \pi$ decay modes of the $a_{4}(2040)$ in a resonancemodel fit not only improves the accuracy of the estimated $a_{4}(2040)$ parameters, but also allows to extract the branching-fraction ratio between these two decay modes. Correcting the measured yields for the unobserved decays to the $\pi^{-} \pi^{0} \pi^{0}$ final state ${ }^{[\mathrm{f}]}$ gives a branching-fraction ratio of $B_{\rho \pi G, f_{2} \pi F}^{a_{4}, \text { corr }}=2.9_{-0.4}^{+0.6}$.

The $t^{\prime}$ spectra of the $a_{4}(2040)$ and the of non-resonant components in the $4^{++}$waves are shown in figure 2 . The $t^{\prime}$ spectra of the $a_{4}(2040)$ component in both waves are constrained by equation (2.5) to have the same shape. The data are in fair agreement with the exponential model in equation (2.4), except for the highest $t^{\prime}$ bin where the model systematically underestimates the data. Our estimate for the slope parameter of $b=9.2_{-0.5}^{+0.8}(\mathrm{GeV} / c)^{-2}$ lies within the range we observe for most of the resonances. The non-resonant components show a steeper falling $t^{\prime}$ spectrum with slopes of $b=14 \pm 4(\mathrm{GeV} / c)^{-2}$ for the $\rho(770) \pi G$ decay and $b=14.5_{-3.7}^{+1.8}(\mathrm{GeV} / c)^{-2}$ for the $f_{2}(1270) \pi F$ decay.

\section{2 $\mathrm{J}^{P C}=\mathbf{1}^{++}$resonances}

The $1^{++} 0^{+} \rho(770) \pi S$ wave contributes about $30 \%$ to the total intensity and is hence the dominant signal in our data. It exhibits a broad peak in the intensity distribution at about $1.2 \mathrm{GeV} / c^{2}$ (see figure $3 \mathrm{a}$ ). This broad peak is described by the $a_{1}(1260)$ component and the non-resonant component, which have approximately similar intensities. The resonance model cannot describe well the details of the intensity spectrum of the $1^{++} 0^{+} \rho(770) \pi S$ wave within the extremely small statistical uncertainties. This is mainly due to the large contribution of the non-resonant component, in combination with our lack of accurate knowledge about the non-resonant shape. This also leads

\footnotetext{
${ }^{[\mathrm{ff}]}$ We correct the yields for the unobserved decays $a_{4}^{-} \rightarrow \rho^{-} \pi^{-}$and $a_{4}^{-} \rightarrow f_{2}^{-} \pi^{-}$to the $\pi^{-} \pi^{0} \pi^{0}$ final state assuming isospin symmetry. We also include the branching fraction of the $f_{2}(1270)$ into $2 \pi$ and corrections of the isospin factor due to self-interference effects (see ref. [2] for details).
} 


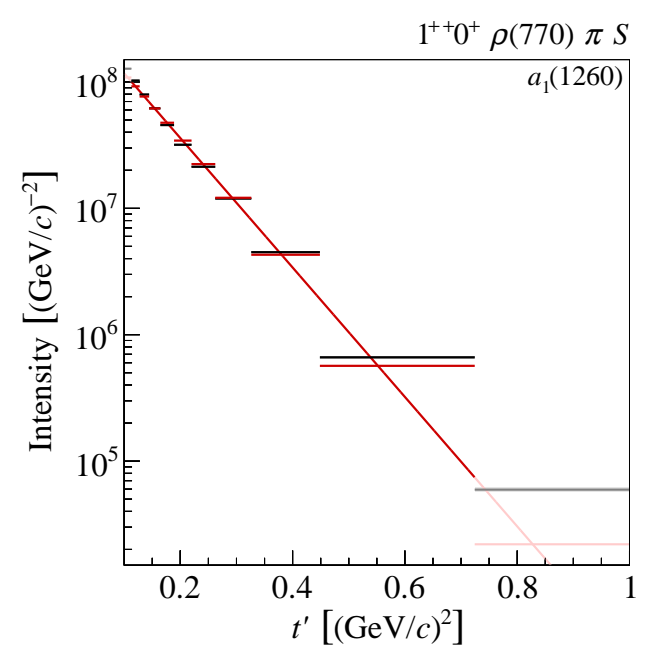

(a)

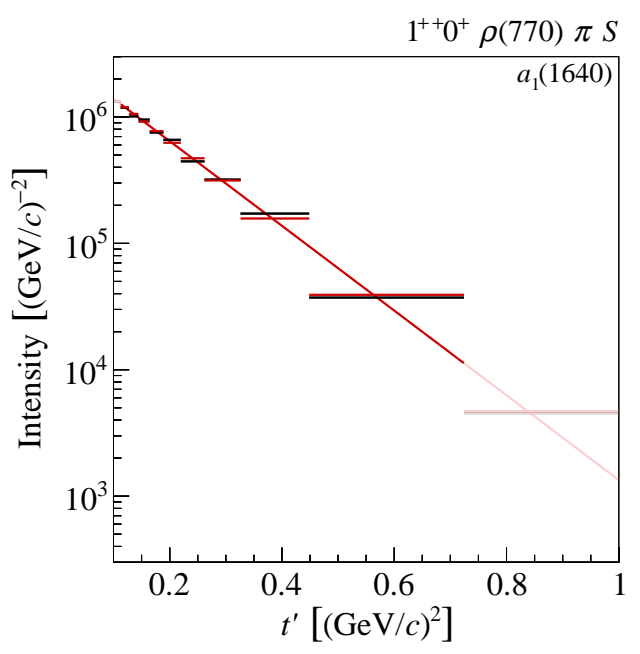

(b)

Figure 4: $t^{\prime}$ spectra of (a) the $a_{1}(1260)$ and (b) the $a_{1}(1640)$ in the $1^{++} 0^{+} \rho(770) \pi S$ wave. Same color code as for the non-resonant components in figure 2 is used.

to large systematic uncertainties of the measured $a_{1}(1260)$ mass and width of $1299_{-28}^{+12} \mathrm{MeV} / c^{2}$ and $380 \pm 80 \mathrm{MeV} / c^{2}$, respectively, which are in agreement with the PDG estimates [9].

We observe evidence for a potential $a_{1}(1640)$ in the high-mass tail of the $a_{1}(1260)$, visible as a shoulder at about $1.8 \mathrm{GeV} / c^{2}$ in the intensity spectrum of the $1^{++} 0^{+} \rho(770) \pi S$ wave. The strongest evidence for the $a_{1}(1640)$ is observed in the $1^{++} 0^{+} f_{2}(1270) \pi P$ wave. It shows a clear peak at about $1.8 \mathrm{GeV} / c^{2}$ (see figure $3 \mathrm{~b}$ ) that is associated with a rising phase motion in this mass region (see figure $3 \mathrm{c}$ ). Both features are reproduced well by the resonance model. Our estimate for the $a_{1}(1640)$ parameters are $m_{0}=1700_{-130}^{+35} \mathrm{MeV} / c^{2}$ and $\Gamma_{0}=510_{-90}^{+170} \mathrm{MeV} / c^{2}$. The PDG lists the $a_{1}(1640)$ as "omitted from summary table" [9]. Our mass value is in agreement the world average, but our estimate for the width is $260 \mathrm{MeV} / c^{2}$ larger than the world average. This might be due to the disagreement between model and data in the $a_{1}(1260)$ mass region or it might be a consequence of not including any higher-lying $a_{1}$ states in the fit model. However, in the analyzed $1^{++}$waves, we do not observer clear evidence for further $a_{1}$ states.

The $t^{\prime}$ spectra of the $a_{1}(1260)$ and the $a_{1}(1640)$ (see figure 4) are in good agreement with the exponential model in equation (2.2). The slope parameter of the $a_{1}(1260)$ of $b=11.8_{-4.2}^{+0.9}(\mathrm{GeV} / c)^{-2}$ is unusually large. The $a_{1}(1260)$ shows the steepest $t^{\prime}$ spectrum among all resonances included in the resonance-model fit. However, it shows a considerable systematic uncertainty towards smaller slope values. The $a_{1}(1260)$ slope value agrees within uncertainties with the slope value of the nonresonant term. This might indicate that the fit is not able to completely separate the $a_{1}(1260)$ from the non-resonant part. The slope parameter of the $a_{1}(1640)$ of $b \approx 8(\mathrm{GeV} / c)^{-2}$ is smaller than the one of the ground state $a_{1}(1260)$ and in the range we typically observe for resonance components.

\section{3 $\mathrm{J}^{P C}=2^{-+}$resonances}

We include four partial waves with $J^{P C}=2^{-+}$in the resonance-model fit, each of which is parameterized by three $\pi_{2}$ resonance components and a non-resonant component. Of the four 


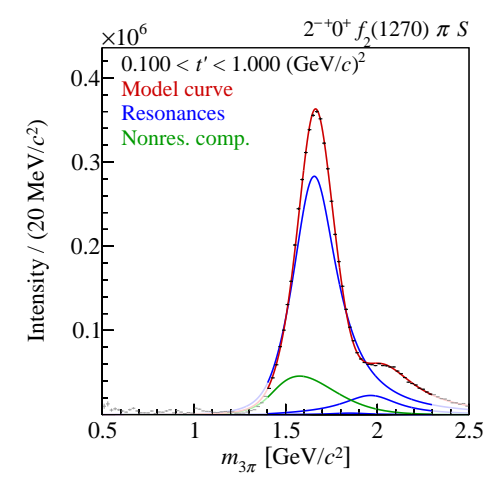

(a)

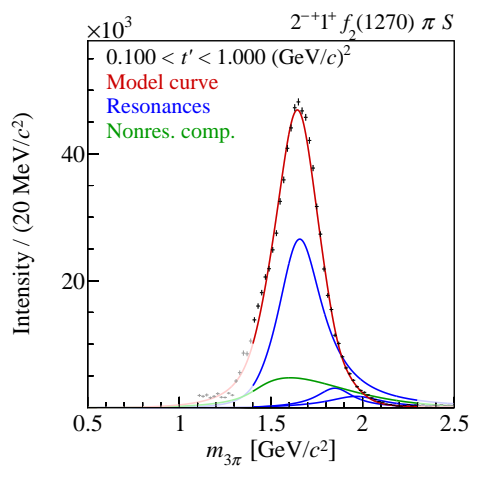

(b)

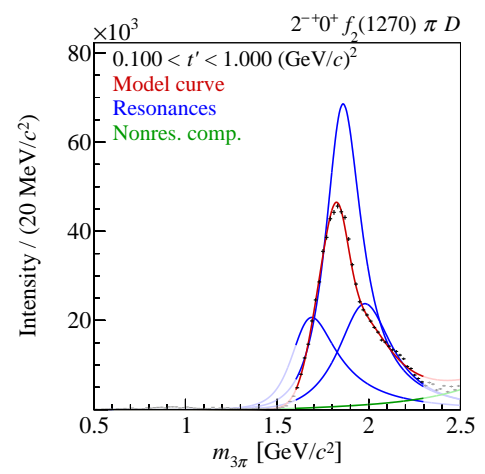

(c)

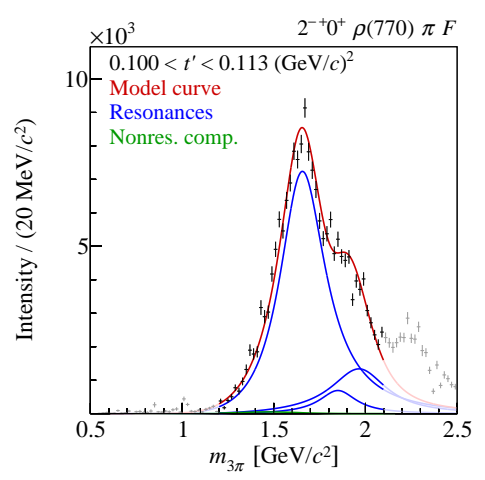

(d)

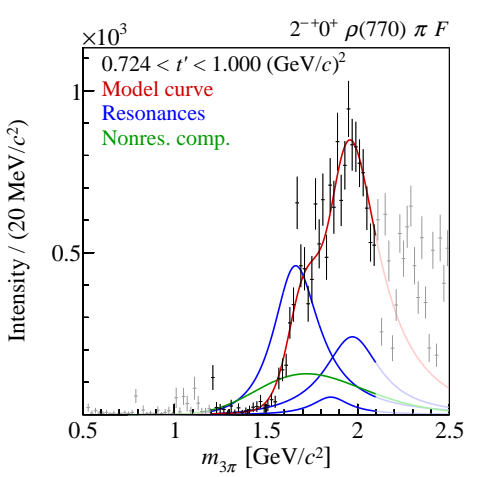

(e)

Figure 5: $t^{\prime}$-summed intensity distributions of (a) the $2^{-+} 0^{+} f_{2}(1270) \pi S$, (b) the $2^{-+} 1^{+}$ $f_{2}(1270) \pi S$, and (c) the $2^{-+} 0^{+} f_{2}(1270) \pi D$ waves. (d) and (e) show the $2^{-+} 0^{+} \rho(770) \pi F$ partial-wave intensity distribution in the lowest and highest $t^{\prime}$ bin, respectively. Same color code as in figure 1 is used.

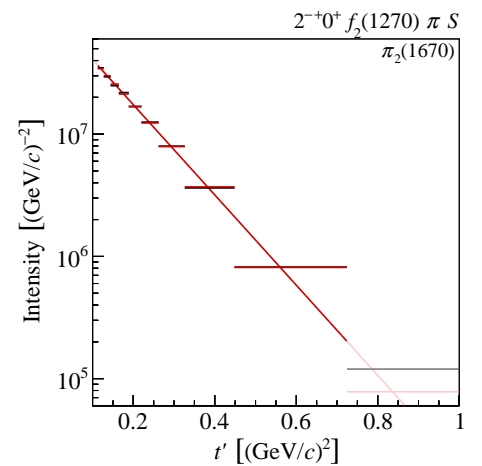

(a)

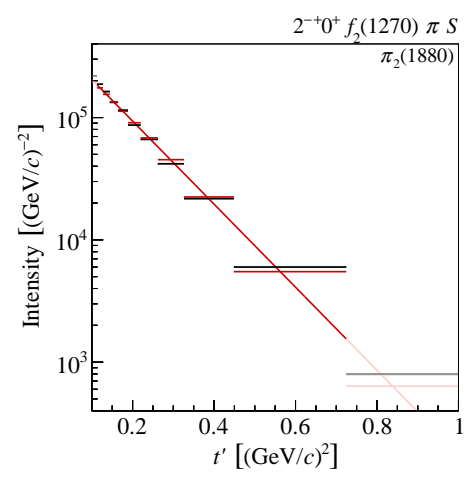

(b)

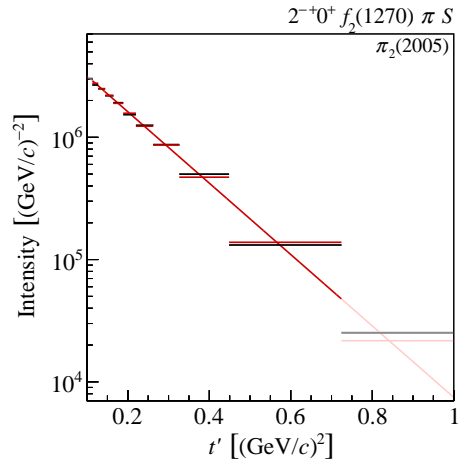

(c)

Figure 6: $t^{\prime}$ spectra of (a) the $\pi_{2}(1670)$, (b) the $\pi_{2}(1880)$, and (c) the $\pi_{2}(2005)$ in the $2^{-+} 0^{+}$ $f_{2}(1270) \pi S$ wave. Same color code as for the non-resonant components in figure 2 is used. 


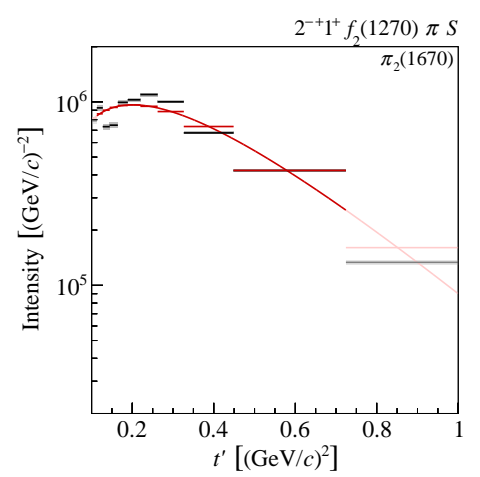

(a)

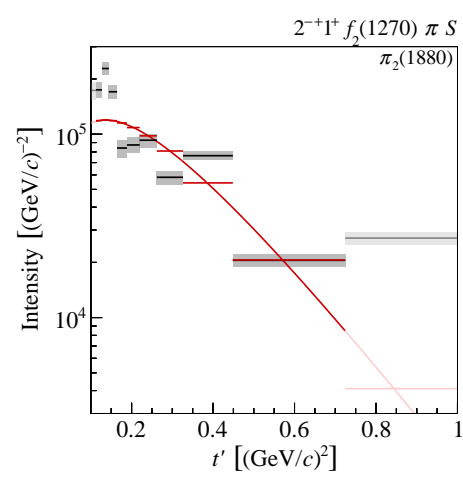

(b)

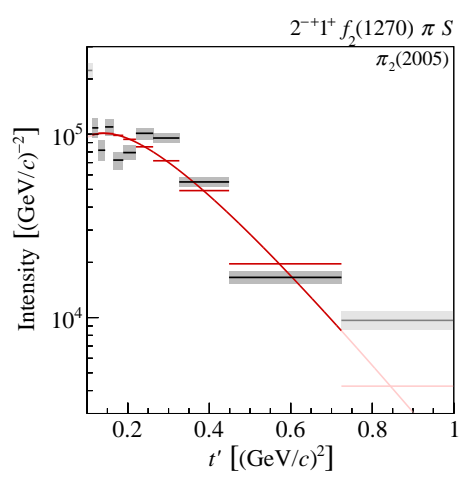

(c)

Figure 7: $t^{\prime}$ spectra of (a) the $\pi_{2}(1670)$, (b) the $\pi_{2}(1880)$, and (c) the $\pi_{2}(2005)$ in the $2^{-+} 1^{+}$ $f_{2}(1270) \pi S$ wave. Same color code as for the non-resonant components in figure 2 is used.

waves, the $2^{-+} 0^{+} f_{2}(1270) \pi S$ wave has the largest intensity. It exhibits a striking peak at about $1.65 \mathrm{GeV} / c^{2}$ (see figure 5a). This peak is reproduced well by a dominant contribution of the $\pi_{2}(1670)$ component. We also include the $2^{-+} 1^{+} f_{2}(1270) \pi S$ wave with spin-projection $M=1$ (see figure $5 \mathrm{~b}$ ), which shows similar features as the $M=0$ wave.

The $2^{-+} 0^{+} f_{2}(1270) \pi D$ wave exhibits a striking peak as well. However, not at $1.65 \mathrm{GeV} / c^{2}$, but at about $1.8 \mathrm{GeV} / c^{2}$ (see figure $5 \mathrm{c}$ ). The peak is described mainly by the $\pi_{2}(1880)$ component. The low- and high-mass tails of the peak are described as an interference effect among the $\pi_{2}(1880)$, the $\pi_{2}(1670)$, and the $\pi_{2}(2005)$ components.

The $2^{-+} 0^{+} \rho(770) \pi F$ wave shows the strongest evidence for the $\pi_{2}(2005)$. In the low- $t^{\prime}$ region (see figure $5 \mathrm{~d}$ ), the intensity spectrum is dominated by a clear peak at about $1.65 \mathrm{GeV} / \mathrm{c}^{2}$, mainly described by the $\pi_{2}(1670)$ component, with a small peak at about $2 \mathrm{GeV} / c^{2}$ in its highmass shoulder. In the high- $t^{\prime}$ region (see figure 5e), the higher-lying peak dominates the intensity spectrum and is mainly described by the $\pi_{2}(2005)$, while the $\pi_{2}(1670)$ is visible only as a low-mass shoulder.

Figure 6 shows the $t^{\prime}$ spectra of the three $\pi_{2}$ resonances in the $2^{-+} 0^{+} f_{2}(1270) \pi S$ wave. They are in good agreement with the exponential model in equation (2.4). The extracted slope parameters of $b=8.5_{-0.5}^{+0.9}(\mathrm{GeV} / c)^{-2}$ for the $\pi_{2}(1670), b=7.8_{-0.9}^{+0.5}(\mathrm{GeV} / c)^{-2}$ for the $\pi_{2}(1880)$, and $b=$ $6.7_{-1.3}^{+0.5}(\mathrm{GeV} / c)^{-2}$ for the $\pi_{2}(2005)$ lie within the range we typically observe for resonances. We observe a similar pattern as for the ground and excited $a_{1}$ states, that the slope parameters decrease for increasing resonance masses. The $t^{\prime}$ spectra of the resonances in the other two $2^{-+}$partial waves with $M=0$ are constrained by equation (2.5) to have the same shapes as the ones in of the $2^{-+} 0^{+}$ $f_{2}(1270) \pi S$ wave. However, in the $2^{-+} 1^{+} f_{2}(1270) \pi S$ wave, the $t^{\prime}$ dependence of the resonance components is independent from the ones in the $M=0$ waves. Figure 7 shows the $t^{\prime}$ spectra of the components in the $2^{-+} 1^{+} f_{2}(1270) \pi S$ wave. In general, the $t^{\prime}$ spectra of the components in this wave are extracted less reliably because: (i) they are constrained by only one wave, while the $M=0$ spectra are constrained by three waves and (ii) the $f_{2}(1270) \pi S$ wave with $M=1$ is small compared to the one with $M=0$. The $t^{\prime}$ spectra for the $M=1$ wave are in rough agreement with the exponential model in equation (2.4). In particular, they exhibit a drop in intensity towards small 
values of $t^{\prime}$ as predicted by the model (see section 2.2). The slope parameters for the $\pi_{2}(1880)$ and the $\pi_{2}(2005)$ in the $M=1$ wave are consistent with the ones in the $M=0$ waves. However, for the $\pi_{2}(1670)$, we estimate a slope parameter of $b \approx 5.0(\mathrm{GeV} / c)^{-2}$ for the $M=1$ wave, which is significantly smaller than for the $M=0$ waves. This effect is not understood, but it is consistent with the shallower $t^{\prime}$ spectrum of the total intensity in the $M=1$ wave.

\section{4. $t^{\prime}$ dependence of relative phases between resonances}

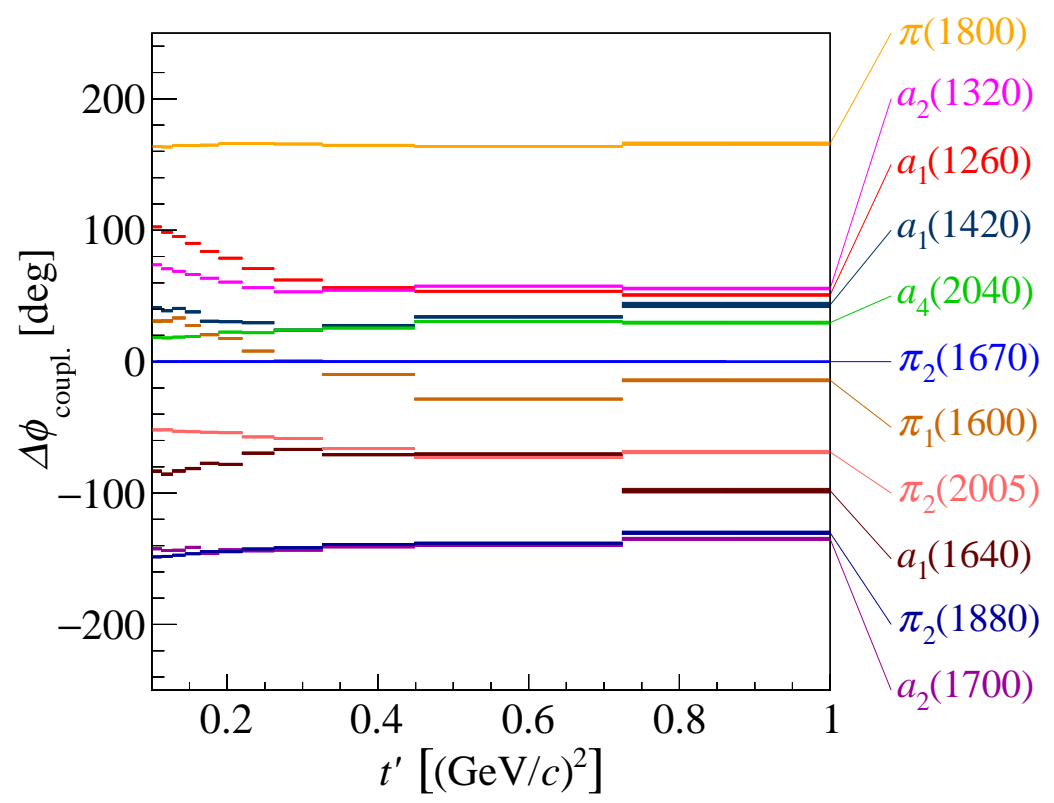

Figure 8: $t^{\prime}$ dependence of the relative phases $\Delta \phi_{\text {coupl. }}$ of the coupling amplitudes of the 11 resonance components in the fit model with respect to the $\pi_{2}(1670)$. The coupling phases are shown for the dominant wave of the respective $J^{P C}$ sector: $0^{-+} 0^{+} f_{0}(980) \pi S, 1^{++} 0^{+} \rho(770) \pi S, 1^{-+} 1^{+}$ $\rho(770) \pi P, 2^{++} 1^{+} \rho(770) \pi D, 2^{-+} 0^{+} f_{2}(1270) \pi S$, and $4^{++} 1^{+} \rho(770) \pi G$. The only exception is the $a_{1}(1420)$, which appears only in the $1^{++} 0^{+} f_{0}(980) \pi P$ wave. The width of the horizontal lines represents the statistical uncertainty. The systematic uncertainty is not shown.

In addition to the $t^{\prime}$ dependence of the intensity of a wave component $j$ in wave $a$, i.e. its $t^{\prime}$ spectrum, we can extract also the $t^{\prime}$ dependence of the relative phase of the coupling amplitude of wave component $j$ in wave $a$ with respect to the wave component $k$ in wave $b$. This is called the coupling phase:

$$
\Delta \phi_{\text {coupl. }}^{j, a ; k, b}\left(t^{\prime}\right) \equiv \arg \left[\mathscr{C}_{a}^{j}\left(t^{\prime}\right) \mathscr{C}_{b}^{k *}\left(t^{\prime}\right)\right]
$$

Figure 8 shows the coupling phases of all 11 resonance components included in the resonance model relative to the $\pi_{2}(1670)$ in the $2^{-+} 0^{+} f_{2}(1270) \pi S$ wave. We observe three striking features of the $t^{\prime}$ dependence of the coupling phases of the resonances. First, for $t^{\prime} \gtrsim 0.3 \mathrm{GeV} / c^{2}$ the phases level off, while for $t^{\prime} \lesssim 0.3 \mathrm{GeV} / c^{2}$ most of the resonances show a slight change of the coupling 


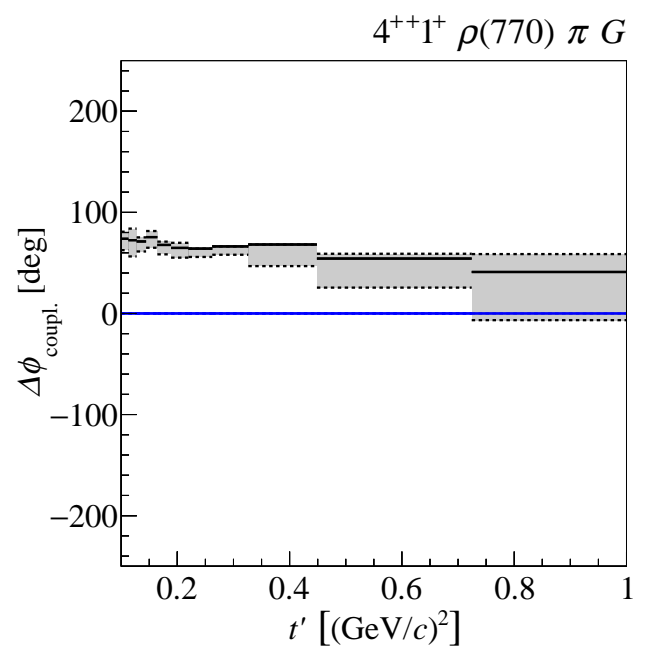

(a)

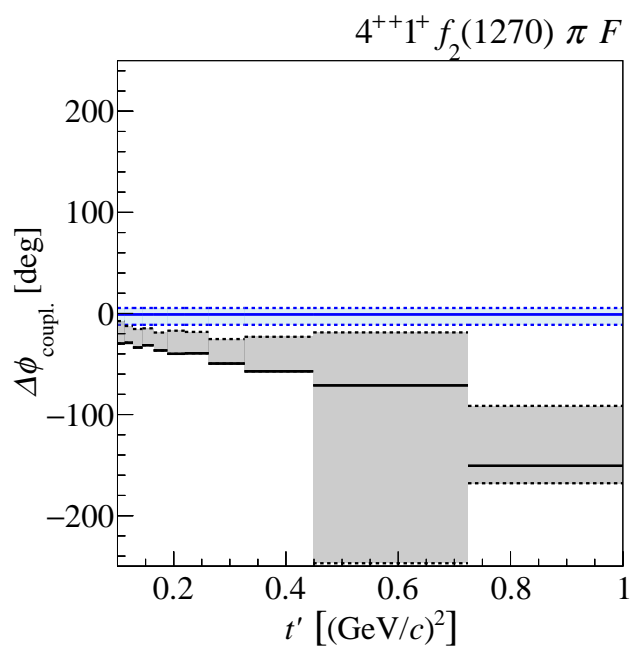

(b)

Figure 9: $t^{\prime}$ dependence of the coupling phase of the wave components in (a) the $4^{++} 1^{+} \rho(770) \pi G$ and (b) the $4^{++} 1^{+} f_{2}(1270) \pi F$ wave. The coupling phases of the $a_{4}(2040)$ (blue lines) and of the non-resonant component (black lines) are shown relative to the $a_{4}(2040)$ in the $4^{++} 1^{+} \rho(770) \pi G$ wave. For each wave component, the magnitude of the effects observed in the systematic studies is illustrated qualitatively by two sets of dashed lines with shaded area in between (see ref. [2] for details).

phase with respect to the $\pi_{2}(1670)$. Second, ground and excited states show large relative phase offsets in the high- $t^{\prime}$ region, with the exception of the $a_{1}(1420)$. The phase of the $a_{1}(1420)$ is very similar to the one of the ground-state $a_{1}(1260)$, which points to the peculiar nature of this signal (see ref. [2] for details). Third, in the high- $t^{\prime}$ region the coupling phases of ground-state resonances do not deviate by more than $\pm 60^{\circ}$ from the phase of the $\pi_{2}(1670)$. These features are consistent with a common production mechanism for the observed signals.

We can also study the coupling phases of the same state in different decay modes. Figure 9 shows the coupling phases of the $a_{4}(2040)$ in the $\rho(770) \pi G$ and $f_{2}(1270) \pi F$ decay modes together with the coupling phases of the corresponding non-resonant components in these waves. As the relative coupling phase of the $a_{4}(2040)$ in the $\rho(770) \pi G$ decay is $0^{\circ}$ by definition, its coupling phase in the $f_{2}(1270) \pi F$ decay has to be a constant as both are related by equation (2.5). However, the model allows an arbitrary phase offset between the two decay modes. We estimate this phase offset to be close to $0^{\circ}$ with small systematic uncertainties. The non-resonant components in the two waves show a slight variation of their coupling phase with $t^{\prime}$ with respect to the $a_{4}(2040)$ resonance. This feature is also observed for the non-resonant components in other partial waves.

\section{Conclusion}

We have performed the so far largest resonance-model fit that simultaneously and consistently describes 14 partial waves including their mutual interferences by a single model. The huge amount of information condensed in this fit, in combination with the information from the our novel $t^{\prime}$ - 
resolved analysis approach allows us to study ground as well as excited states. It also allows us to study in detail the $t^{\prime}$ dependence of the intensity and coupling phase of these resonances. We observe that for most partial waves, the $t^{\prime}$ spectra of the non-resonant components fall steeper with $t^{\prime}$ as compared to the resonances. This helps to separate resonant from non-resonant contributions. We also observe that for most of the resonances, the $t^{\prime}$ spectra become shallower with increasing resonance mass. This is consistent with the observation that the slope of the overall $t^{\prime}$ spectrum of the data becomes shallower for higher $m_{3 \pi}$ masses (see ref. [1]). Furthermore, the different $t^{\prime}$ dependences of the ground-state and the excited resonances helps to better separate them. An example is the strong evolution of the intensity spectrum of the $2^{-+} 0^{+} \rho(770) \pi F$ wave with $t^{\prime}$ (see figures $5 \mathrm{~d}$ and 5e). Our approach also allows to study the $t^{\prime}$ dependence of the relative phases of the resonances. The pattern we observe is consistent with a common production mechanism. To reduce the systematic uncertainties and to further clarify the existence of higher excited states, models that are based on the principles of unitarity and analyticity are mandatory, especially in waves where the non-resonant contributions are important. This is the topic of future research.

\section{References}

[1] C. Adolph et al. [COMPASS Collaboration], "Resonance production and $\pi \pi S$-wave in $\pi^{-}+p \rightarrow \pi^{-} \pi^{-} \pi^{+}+p_{\text {recoil }}$ at $190 \mathrm{GeV} / c$ ", Physical Review D 95 (2017), 032004, DOI: 10.1103 /PhysRevD . 95.032004.

[2] R. Akhunzyanov et al. [COMPASS Collaboration], "Light isovector resonances in $\pi^{-} p \rightarrow \pi^{-} \pi^{-} \pi^{+} p$ at $190 \mathrm{GeV} / c^{\prime}$, (2018), arXiv: 1802.05913.

[3] M. L. Perl, High energy hadron physics, Wiley, 1974, ISBN: 0-471-68049-4.

[4] M. Alekseev et al. [COMPASS Collaboration], "Observation of a $J^{P C}=1^{-+}$Exotic Resonance in Diffractive Dissociation of $190 \mathrm{GeV} / c \pi^{-}$into $\pi^{-} \pi^{-} \pi^{+}$, Phys. Rev. Lett. 104 (2010), 241803, DOI: 10 .1103/PhysRevLett. 104.241803.

[5] C. Adolph et al. [COMPASS Collaboration], "Odd and even partial waves of $\eta \pi^{-}$and $\eta^{\prime} \pi^{-}$in $\pi^{-} p \rightarrow \eta^{(\prime)} \pi^{-} p$ at $191 \mathrm{GeV} / c^{\prime}$, Phys. Lett. B 740 (2015), 303-311, DOI: $10.1016 /$ j.phys letb. 2014.11 .058$.

[6] S. U. Chung et al. [E852 collaboration], "Exotic and $q \bar{q}$ resonances in the $\pi^{+} \pi^{-} \pi^{-}$system produced in $\pi^{-} p$ collisions at $18 \mathrm{GeV} / c$ ", Phys. Rev. D 65 (2002), 072001, DoI: 10.1103 /PhysRevD . 65.072001.

[7] D. V. Amelin et al. [VES Collaboration], "Investigation of the reaction $\pi^{-}+A \rightarrow \omega \pi^{-} \pi^{0}+A^{*}$ ", Phys. Atom. Nucl. 62 (1999), [Yad. Fiz. 62, 487 (1999)], 445-453.

[8] A. V. Anisovich et al., "Partial wave analysis of $\bar{p} p$ annihilation channels in flight with $I=1, C=+1$ ", Phys. Lett. B 517 (2001), 261-272, DOI: $10.1016 /$ S0370-2693 (01) 01017-6.

[9] C. Patrignani et al. [Particle Data Group], "Review of Particle Physics", Chin. Phys. C 40 (2016), 100001, DOI: 10.1088/1674-1137/40/10/100001. 